\title{
Small scale isocurvature perturbation of WIMP dark matter
}

\section{Ki-Young CHOI*}

Department of Physics, Chonnam National University, 77 Yongbong-ro, Buk-gu, Gwangju, 61186, Republic of Korea

E-mail: kiyoungchoi@jnu.ac.kr

\section{Jinn-Ouk Gong}

Asia Pacific Center for Theoretical Physics, Pohang, 790-784, Korea

Department of Physics, Postech, Pohang 790-784, Korea

E-mail: jinn-ouk.gongeapctp.org

\section{Chang Sub Shin}

Department of Physics and Astronomy, Rutgers University, Piscataway NJ 08854, USA

E-mail: changsub@physics.rutgers.edu

\begin{abstract}
The smallest size of the cosmic structures is known to be limited in the standard dark matter models with weakly interacting massive particles. However this constraint can be relaxed in the low reheating temperature scenarios. During the transition from the early matter domination to the radiation domination, the small scale isocurvature perturbation of WIMP is created and become large. These are not erased during the kinetic decoupling and can form the small size structures such as ultra compact mini halos. Combining with the WIMP properties, the small objects can show their existence with visible signatures like gamma ray, cosmic ray or neutrinos.
\end{abstract}

11th International Workshop Dark Side of the Universe 2015

14-18 December 2015

Yukawa Institute for Theoretical Physics, Kyoto University Japan

${ }^{*}$ Speaker. 


\section{Introduction}

The weakly interacting massive particles (WIMPs) are a natural candidate for dark matter [1]. The nature of the weak interaction and the mass provides the right relic density for dark matter when they freeze out of the thermal equilibrium [2,3]. The same properties are used to search for dark matter at colliders such as Large Hadron Collider at CERN and in the direct detection of dark matter deep under the ground or in the astrophysical phenomena.

The scatterings of the WIMPs that keep them in the state of the thermal equilibrium in the early Universe can be divided into two categories: the inelastic scatterings and the elastic scatterings. Through inelastic scatterings, the WIMPs are changing their numbers as well as exchanging energy and momentum with the background plasma. Therefore the WIMPs can follow the statistics in the thermal equilibrium. Eventually due to the expansion of the Universe, the interactions become insufficient and the WIMPs decouple from the thermal background, at which epoch the relic density of the WIMP dark matter is fixed. The chemical decoupling is commonly happens at a temperature below the WIMP mass around $T \sim m / 20$ with WIMP mass $m$.

However even after the chemical freeze-out, the elastic scatterings continue with exchanging momentum and energy but without changing the number of WIMPs. During this kinetic equilibrium, the WIMPs are still tightly coupled to the background plasma and move together so that their density perturbations evolve in the same way as those of radiation. They oscillate in the subhorizon scales and cannot grow since they are in kinetic equilibrium with radiation. Only after the kinetic decoupling, the perturbations of WIMPs can grow logarythmically in the radiation-dominated Universe. The perturbations in the smaller scales get even damped during the kinetic decoupling, and thus density perturbation becomes too small to make any visible structures in the present Universe. Thus the kinetic decoupling scale puts the smallest size of the cosmic structures in the WIMP dark matter scenarios.

As studied in [4], the kinetic decoupling temperature of the usual neutralinos is between 10 $\mathrm{MeV}$ and several $\mathrm{GeV}$ for the mass of neutralinos between $10 \mathrm{GeV}$ and several TeV. In other words, this temperature corresponds to the mass scale from $10^{-10}$ to $10^{-4}$ solar mass and the earth mass size is located between them. The structures with smaller size than the this minimum cannot form in the WIMP dark matter models.

However there is an exceptional case where the smaller size structures can form even during the kinetic equilibrium in the WIMP dark matter case, that seems to be conflict with the previous one. The new phenomena was studied recently by Choi, Gong and Shin [5]. In the study, the large isocurvature perturbations at small scales can arise during the transition from the early matter domination to the radiation domination and these perturbations are not damped away during the kinetic decoupling. Therefore the small scale structures made of dominantly WIMPs may exist in the present Universe and produce new visible signatures.

In this talk, we briefly review the evolution of the WIMP perturbation and the possible generation of the large isocurvature perturbation of them at small scales in the low reheating temperature scenario. In the end, we summarise with the implications of this phenomena and prospects in the future observations. 


\section{Adiabatic and Isocurvature Perturbation of WIMP}

The isocurvature perturbation between dark matter and radiation is defined as

$$
S \equiv 3 H\left(\frac{\delta \rho_{m}}{\dot{\rho}_{m}}-\frac{\delta \rho_{r}}{\dot{\rho}_{r}}\right)=\delta_{m}-\frac{3}{4} \delta_{r}
$$

On large scales, temperature anisotropy of the cosmic microwave background constrains this mode to be smaller than the adiabatic mode by a few percent [6].

Since WIMPs are in the thermal equilibrium with background radiation in the early Universe, their density perturbation follows those of the radiation. Therefore two fluids of dark matter and radiation have the common density perturbations, that is the nature of adiabaticity. The WIMP dark matters are thus inherently adiabatic on large scales.

However after decoupling, it is not necessary to have common perturbation. They would have different evolutions in the subhorizon scales and the isocurvature perturbation can be generated. That is not problematic since the isocurvature perturbation on small scales is not constrained well enough.

For WIMPs, the evolutions of the modes are different whether they enter the horizon before or after the kinetic decoupling. For the small modes that enter the horizon before kinetic decoupling, they experience damping and the perturbations become quite suppressed. Only after the kinetic decoupling, they could grow logarythmically during radiation domination from the suppressed amplitude. The modes, that enter the horizon after the kinetic decoupling, do not have the suppression and could grow from the initial amplitude of the perturbation. Those modes finally become the seeds for the large scale structure formation after the matter-radiation equality.

The non-vanishing isocurvature perturbation between dark matter and radiation is generated after the kinetic decoupling since the evolutions of dark matter and radiation are now separated. Though the isocurvature perturbation in the literature usually refer to the mode in the primordial perturbation on large scales, here also we use it on small scales too on general grounds as a function of time.

\section{Large Isocurvature Perturbation of WIMP with Low Reheating Temperature}

The question is whether it is possible to have a large isocurvature perturbation at small scales even before the kinetic decoupling without being damped. The answer is 'Yes' when the rehearing temperature is low enough than the freeze-out temperature of WIMP [5]. There are two facts we have to note in this process: one is the creation of the isocurvature perturbation and the other is non-damping of them.

The latter part was used in the literature to explain 'the cosmic background temperature anisotropy in a minimal isocurvature model for galaxy formation' by P.J.E. Peebles [7]. While the adiabatic modes are damped during the kinetic decoupling, however the isocurvature mdoes are not affected. The same is true for dark matter. When there is an isocurvature perturbations in the dark matter density, those are not damped during the kinetic decoupling and continue to remain. If initially it was large enough then it could make new contributions to the formation of small scale objects. 
One example for the creation of large isocurvature perturbation was found in [5]: when the reheating temperature after inflation is lower than the freeze-out temperature of WIMP. After inflation, the inflaton field oscillates around the vacuum and the Unvierse is like matter-dominated. Due to the continuous decay of the inflaton field, the radiation and WIMPs are created. Here the maximum temperature can be still high enough so that the WIMPs are in the thermal equilibrium before freeze-out. Therefor on large scales the WIMP density perturbation is intrinsically adiabatic. However on small scales it makes difference. After chemical freeze-out during matter-domination, the WIMPs are not created any more and the comoving number density is preserved. However the radiation is continuously produced from the decay of inflaton field until they dominated the energy density of the Universe. Here we assume that the dark matter is not produced directly from the decay of inflaton. During this epoch the isocurvature perturbation between dark matter and radiation is created due to the different evolution of the number densities of the radiation and dark matter.

The generated isocurvature perturbation is roughly estimated by [5]

$$
\delta_{m}\left(t_{\mathrm{reh}}\right) \simeq \frac{5}{4} \Phi_{0}\left(\frac{k}{k_{\mathrm{reh}}}\right)^{2}, \quad \text { for } \quad k>k_{\text {reh }}
$$

where $\delta_{m}\left(t_{\mathrm{reh}}\right)$ is $\delta \rho_{m} / \rho_{m}$ at the time of reheating and $\Phi_{0}$ is the gravitational potential at initial time with the corresponding scale $k$ of interest compared to the scale $k_{\text {reh }}$ at the reheating time. For the modes which enter the horizon before reheating $\left(k>k_{\text {reh }}\right)$, there is enhancement from the initial density contrast proportional to the squared of the scales. This perturbation becomes isocurvature and does not have damping afterwards and continues until the matter-radiation equality.

During matter domination, this large perturbations quickly form the compact mini structures and they may exist afterwards until now to produce visible signals. For example, in the Milky Way halo there may exist compact minihalos of earth size or even smaller mostly dominated by WIMP dark matter and they annihilate to produce gamma rays or cosmic rays. Those signatures are well probed by the Fermi-LAT gamma-ray telescope or AMS-02 cosmic ray telescope.

\section{Summary}

In the standard WIMP dark matter scenario with high reheating temperature, the kinetic decoupling scale puts the lower bound on the smallest size of the structures in the Universe. For very weakly interacting particles like gravitino or axino [8], there is no lower bound on the smallest size. However at the same time, due to the very weak interactions, they don't make any visible signatures. However WIMP dark matter may annihilate and produce many visible effects if they can make large number of small scale objects. In the low-reheating temperature scenarios, it is possible that the WIMP dark matter can obtain large isocurvature perturbation and form the small scale structures dominated by WIMP. These WIMP-dominated objects can make visible signatures in the present Universe.

\section{References}

[1] H. Baer, K. Y. Choi, J. E. Kim and L. Roszkowski, Phys. Rept. 555 (2014) 1 doi:10.1016/j.physrep.2014.10.002 [arXiv:1407.0017 [hep-ph]]. 
[2] P. Hut, Phys. Lett. B 69, 85 (1977). doi:10.1016/0370-2693(77)90139-3

[3] B. W. Lee and S. Weinberg, Phys. Rev. Lett. 39 (1977) 165. doi:10.1103/PhysRevLett.39.165

[4] T. Bringmann, New J. Phys. 11 (2009) 105027 doi:10.1088/1367-2630/11/10/105027 [arXiv:0903.0189 [astro-ph.CO]].

[5] K. Y. Choi, J. O. Gong and C. S. Shin, Phys. Rev. Lett. 115 (2015) no.21, 211302 doi:10.1103/PhysRevLett.115.211302 [arXiv:1507.03871 [astro-ph.CO]].

[6] P. A. R. Ade et al. [Planck Collaboration], arXiv:1502.01589 [astro-ph.CO].

[7] P. J. E. Peebles, ApJ, 315:L73 (1987).

[8] K. Y. Choi, L. Covi, J. E. Kim and L. Roszkowski, JHEP 1204 (2012) 106 doi:10.1007/JHEP04(2012)106 [arXiv:1108.2282 [hep-ph]]. 